\title{
Pasta de higuerilla desintoxicada en dietas para pollos de engorda
}

Anabel Maldonado Fuentes ${ }^{a}$

Juan Manuel Cuca García ${ }^{\text {a }}$

Arturo Pro Martínez a*

Fernando González Cerón ${ }^{b}$

José Guadalupe Herrera Haro ${ }^{a}$

Eliseo Sosa Montes ${ }^{b}$

Pablo Alfredo Domínguez Martínez ${ }^{\mathrm{c}}$

${ }^{a}$ Colegio de Postgraduados, Campus Montecillo, PRGP- Ganadería, Estado de México, México.

${ }^{\mathrm{b}}$ Universidad Autónoma Chapingo, Departamento de Zootecnia, Estado de México, México.

${ }^{\mathrm{c}}$ Instituto Nacional de Investigaciones Forestales, Agrícolas y Pecuarias, Departamento de Producción Animal, Estado de Durango, México.

*Autor de correspondencia: aproma@colpos.mx

\section{Resumen:}

La pasta de higuerilla (Ricinus communis L) contiene sustancias altamente tóxicas, por lo que se evaluó la efectividad de tres métodos de desintoxicación y su inclusión en dietas para pollos de engorda. Se evaluaron cinco tratamientos (dietas experimentales): dieta testigo a base de maíz y pasta de soya (PS), pasta de higuerilla sin desintoxicar (PH), pasta de higuerilla tratada con autoclave (PHA), pasta de higuerilla tratada con el método químico (PHQ) y pasta de higuerilla tratada con autoclave y método químico (PHAQ). Cada tratamiento se asignó al azar a siete unidades experimentales con 10 pollos cada una. Las variables evaluadas fueron: consumo de alimento (COA), conversión alimenticia (CA), ganancia de peso (GP) rendimiento en canal (RC), rendimiento de pechuga (RP), rendimiento de pierna con muslo (RPM), desarrollo del 
sistema digestivo, habilidad para caminar (HC), angulación valgus /varus (AVV) y latencia a postrarse (LP). Los pollos alimentados con PH y PHMQ tuvieron menor COA y GP $(P<0.05)$, sin embargo, no hubo diferencias entre tratamientos para CA. Se encontraron diferencias entre tratamiento $(P<0.05)$ para HC y AVV, mientras que en LP no hubo diferencias $(P>0.05)$. Los resultados evidencian que el tratamiento con autoclave (1 atmosfera de presión durante $60 \mathrm{~min}$ a $121^{\circ} \mathrm{C}$ ) disminuyó la toxicidad en la pasta de higuerilla, ya que las aves del tratamiento PHMA tuvieron un comportamiento productivo similar $(P>0.05)$ a las de la dieta testigo.

Palabras clave: Ricinus communis L., Métodos de desintoxicación, Autoclave, Hidróxido de calcio, Pollos de engorda.

Recibido: 29/08/2018

Aceptado: 28/08/2019

\section{Introducción}

La higuerilla o ricino (Ricinus communis L) es una planta originaria de África perteneciente a la familia Euphorbiaceae, se encuentra distribuida por todo el mundo, principalmente en India, China y Brasil; se destaca por su rusticidad, la tolerancia a la sequía y al alto contenido de aceite en sus semillas ${ }^{(1)}$, en México hay condiciones agroecológicas propicias para el cultivo de higuerilla en el sur y sureste principalmente $^{(2)}$. El aceite de ricino se ha utilizado para la producción de biodiesel y como subproducto de su extracción se obtiene la pasta de higuerilla que corresponde aproximadamente al $55 \%$ del peso de la semilla ${ }^{(3)}$.

La composición nutricional de la pasta de higuerilla ${ }^{(4,5)}$, indica que es posible la inclusión en la alimentación animal, ya que puede ser una alternativa en la sustitución de ingredientes proteínicos y disminuir los costos de producción. Pero su utilización está limitada debido a la presencia de productos tóxicos y alérgenos, principalmente, ricina, ricinina, y el alérgeno $\mathrm{CB}-1 \mathrm{~A}$, siendo la ricina la más tóxica ${ }^{(6)}$. Sin embargo, actualmente hay métodos eficientes para desintoxicar los subproductos de semillas de ricino y están enfocados en la disminución o eliminación de la ricina, el calor con presión en autoclave y los tratamientos con hidróxido de calcio son eficientes en la eliminación de los compuestos tóxicos ${ }^{(7,8)}$; la cocción de la semilla, además de la fermentación en agua, disminuyen la toxicidad de la pasta de higuerilla y permiten su inclusión en las dietas para aves sin afectar el comportamiento productivo ${ }^{(9,10)}$. 
La ricina se inactiva a altas temperaturas y en álcalis fuertes; al respecto Anandan et $a l^{(7)}$ no encontraron residuos de ricina en muestras de pasta de higuerilla tratada con autoclave (1 atm de presión y $121{ }^{\circ} \mathrm{C}$ por $60 \mathrm{~min}$ ) o con hidróxido de calcio (40 g/ $\mathrm{kg}$ ) analizadas mediante electroforesis en gel de poliacrilamida. De esta forma la combinación de estos métodos podría potencializar su efecto en la inactivación de los compuestos tóxicos de la higuerilla; además, no se han realizado estudios donde se incluya pasta de higuerilla tratada por estos métodos en la alimentación de pollos de engorda. Por lo tanto, la hipótesis de este trabajo fue que el uso de los métodos de autoclave (una atmósfera de presión a $121^{\circ} \mathrm{C}$ por $60 \mathrm{~min}$ ), tratamiento químico (40 g de $\left.\mathrm{Ca}(\mathrm{OH})_{2} / \mathrm{kg}\right)$ de pasta de higuerilla o la combinación de éstos en la pasta de higuerilla, permitirán su inclusión en dietas para pollos de engorda, sin afectar las variables productivas y de bienestar animal. De esta forma, el objetivo del presente estudio fue evaluar el efecto de los métodos de autoclave, tratamiento químico o combinación de éstos en el comportamiento productivo y variables de bienestar en pollos de engorda.

\section{Material y métodos}

\section{Desintoxicación de la pasta de higuerilla}

Para la desintoxicación de la pasta de higuerilla se emplearon tres métodos descritos por Anandan et al ${ }^{(7)}$ : método de autoclave (A), método químico con $\mathrm{Ca}(\mathrm{OH})_{2}(\mathrm{Q})$ y combinación de métodos de autoclave con químico (AQ).

\section{Método de autoclave}

Se utilizaron 40 muestras de pasta de higuerilla de 1,000 g cada una, las cuales se introdujeron en una autoclave Felisa, aplicando una atmósfera de presión, durante 60 min a $121^{\circ} \mathrm{C}$. Posteriormente, se secaron al sol por $48 \mathrm{~h}$ y se almacenaron a temperatura ambiente $^{(7)}$.

\section{Método químico con hidróxido de calcio $\mathrm{Ca}(\mathrm{OH})_{2}$}

Veinte (20) muestras de pasta de higuerilla de 1,000 g cada una se mezclaron con hidróxido de calcio a una concentración de $40 \mathrm{~g} / \mathrm{kg}$, se dejaron durante $8 \mathrm{~h}$ por la noche $\mathrm{y}$ posteriormente se secaron al sol durante $48 \mathrm{~h}$, se molieron con un molino manual 
(Estrella, México) y se almacenaron a temperatura ambiente. El hidróxido de calcio fue diluido en agua, antes de ser mezclado con la pasta de higuerilla ${ }^{(7)}$.

\section{Combinación de métodos de autoclave con químico}

Se emplearon los métodos de autoclave e hidróxido de calcio descritos por Anandan et $a l^{(7)}$. Brevemente, después de haber tratado la pasta de higuerilla por el método de autoclave, se aplicó el método de hidróxido de calcio.

\section{Aves y tratamientos}

El experimento se realizó en las instalaciones avícolas del Colegio de Postgraduados, Campus Montecillo, Texcoco, Estado de México. Ubicado a una altitud de 2,247 msnm $^{(11)}$. Se evaluaron cinco tratamientos (dietas experimentales): dieta testigo maíz y pasta de soya (PS), pasta de higuerilla sin desintoxicar (PH), pasta de higuerilla tratada con autoclave (PHMA), pasta de higuerilla tratada con el método químico (PHMQ) y pasta de higuerilla tratada con autoclave y método químico (PHMAQ). Cada tratamiento se asignó al azar a 7 unidades experimentales con 10 pollos cada una. Las aves se alojaron en corrales de $1.5 \mathrm{~m}^{2}$ con cama de viruta de madera. Se proporcionó un régimen de iluminación $23 \mathrm{~h}$ luz durante las primeras dos semanas y posteriormente se disminuyó a $12 \mathrm{~h}$. La temperatura ambiental fue de $33{ }^{\circ} \mathrm{C}$ al inicio del experimento, la cual se redujo $2{ }^{\circ} \mathrm{C}$ por semana hasta llegar a una temperatura de $21^{\circ} \mathrm{C}$. Este estudio se realizó de acuerdo con la Guía de Cuidado y Uso de Animales Experimentales aprobada por el Consejo Académico General del Colegio de Postgraduados.

El programa de alimentación se dividió en dos fases: dieta de iniciación (1-21 días) que contenía: 3,025 kcal de energía metabolizable (EM) $\mathrm{kg}^{-1}, 22 \%$ de proteína cruda (PC), $0.96 \%$ de $\mathrm{Ca}$ y $0.48 \%$ de $\mathrm{P}$ disponible y dieta de finalización (22-42 días) que contenía: 3,100 kcal de EM kg-1, 19 de PC, $0.80 \%$ de Ca y $0.40 \%$ de P disponible (Cuadro 1). Las dietas se formularon para cubrir o exceder las recomendaciones nutricionales de la línea Ross $308^{(12)}$. 
Cuadro 1: Composición de las dietas experimentales para pollos de engorda

\begin{tabular}{|c|c|c|c|c|c|c|c|c|c|c|}
\hline \multirow[b]{2}{*}{ Ingredientes (\%) } & \multicolumn{4}{|c|}{ Iniciación (1-21 días) } & \multicolumn{6}{|c|}{ Finalización (22-42 días) } \\
\hline & PS & PH & PHA & PHQ & $\begin{array}{l}\text { PHA } \\
\mathbf{Q}\end{array}$ & PS & PH & PHA & PHQ & $\begin{array}{l}\text { PHA } \\
\text { Q }\end{array}$ \\
\hline \multirow{2}{*}{ Pasta de soya } & 35.4 & 33.3 & 33.3 & 33.3 & \multirow{2}{*}{33.34} & 30.4 & 28.3 & 28.3 & 28.3 & \multirow{2}{*}{28.36} \\
\hline & 1 & 4 & 4 & 4 & & 4 & 6 & 6 & 6 & \\
\hline \multirow{2}{*}{ Maíz } & 56.2 & 53.9 & 53.9 & 54.0 & \multirow{2}{*}{54.08} & 61.9 & 59.5 & 59.5 & 59.7 & \multirow{2}{*}{59.70} \\
\hline & 9 & 4 & 4 & 8 & & 1 & 6 & 6 & 0 & \\
\hline Pasta de higuerilla & 0.00 & 4.16 & 4.16 & 4.13 & 4.13 & 0.00 & 4.16 & 4.16 & 4.13 & 4.13 \\
\hline Carbonato de calcio & 1.25 & 1.22 & 1.22 & 1.16 & 1.16 & 1.07 & 1.04 & 1.04 & 0.98 & 0.98 \\
\hline Fosfato dicálcico & 2.03 & 2.06 & 2.06 & 2.06 & 2.06 & 1.61 & 1.64 & 1.64 & 1.64 & 1.64 \\
\hline L-lisina & 0.32 & 0.36 & 0.36 & 0.36 & 0.36 & 0.12 & 0.17 & 0.17 & 0.17 & 0.17 \\
\hline DL-metionina & 0.49 & 0.49 & 0.49 & 0.49 & 0.49 & 0.34 & 0.34 & 0.34 & 0.34 & 0.34 \\
\hline L-treonina & 0.13 & 0.14 & 0.14 & 0.14 & 0.14 & 0.02 & 0.03 & 0.03 & 0.03 & 0.03 \\
\hline L-triptófano & 0.00 & 0.00 & 0.00 & 0.00 & 0.00 & 0.00 & 0.00 & 0.00 & 0.00 & 0.00 \\
\hline Aceite & 3.43 & 3.64 & 3.64 & 3.60 & 3.60 & 3.49 & 3.70 & 3.70 & 3.66 & 3.66 \\
\hline Coccidiostato & 0.05 & 0.05 & 0.05 & 0.05 & 0.05 & 0.05 & 0.05 & 0.05 & 0.05 & 0.05 \\
\hline Pigmento & 0.00 & 0.00 & 0.00 & 0.00 & 0.00 & 0.35 & 0.35 & 0.35 & 0.35 & 0.35 \\
\hline Sal & 0.30 & 0.30 & 0.30 & 0.30 & 0.30 & 0.30 & 0.30 & 0.30 & 0.30 & 0.30 \\
\hline $\begin{array}{l}\text { Vitaminas y } \\
\text { minerales* }\end{array}$ & 0.30 & 0.30 & 0.30 & 0.30 & 0.30 & 0.30 & 0.30 & 0.30 & 0.30 & 0.30 \\
\hline Total & 100 & 100 & 100 & 100 & 100 & 100 & 100 & 100 & 100 & 100 \\
\hline \multicolumn{11}{|c|}{ Análisis calculado (\%) } \\
\hline Proteína cruda & 21.0 & 21.0 & 21.0 & 21.0 & 21.0 & 19.0 & 19.0 & 19.0 & 19.0 & 19.0 \\
\hline EM, kcal & 3025 & 3025 & 3025 & 3025 & 3025 & 3100 & 3100 & 3100 & 3100 & 3100 \\
\hline Calcio & 0.96 & 0.96 & 0.96 & 0.96 & 0.96 & 0.80 & 0.80 & 0.80 & 0.80 & 0.40 \\
\hline Fósforo disponible & 0.48 & 0.48 & 0.48 & 0.48 & 0.48 & 0.40 & 0.40 & 0.40 & 0.40 & 0.40 \\
\hline Lisina & 1.44 & 1.44 & 1.44 & 1.44 & 1.44 & 1.15 & 1.15 & 1.15 & 1.15 & 1.15 \\
\hline Metionina & 0.83 & 0.83 & 0.83 & 0.83 & 0.83 & 0.47 & 0.66 & 0.66 & 0.66 & 0.66 \\
\hline Metionina+cistina & 1.08 & 1.08 & 1.08 & 1.08 & 1.08 & 0.90 & 0.90 & 0.90 & 0.90 & 0.90 \\
\hline Treonina & 0.97 & 0.97 & 0.97 & 0.97 & 0.97 & 0.78 & 0.78 & 0.78 & 0.78 & 0.78 \\
\hline Triptófano & 0.30 & 0.30 & 0.30 & 0.30 & 0.30 & 0.18 & 0.27 & 0.27 & 0.27 & 0.27 \\
\hline
\end{tabular}

$\mathrm{PS}=$ testigo, dieta base soya-maíz; $\mathrm{PH}=$ dieta con higuerilla sin desintoxicar; $\mathrm{PHA}=$ dieta con higuerilla tratada con método de autoclave; $\mathrm{PHQ}=$ dieta con higuerilla tratada con método químico; $\mathrm{PHAQ}=$ dieta con higuerilla tratada con autoclave y con químico.

*Premezcla de vitaminas por kilogramo de alimento: A, 12,000 UI; $\mathrm{D}_{3}$, 1,000 UI; E, $60 \mathrm{UI} ; \mathrm{K}, 5.0 \mathrm{mg}$; $\mathrm{B}_{2}$, $8.0 \mathrm{mg}$; B $12,0.030 \mathrm{mg}$; ácido pantoténico, $15 \mathrm{mg}$; niacina, $50 \mathrm{mg}$; ácido fólico, $1.5 \mathrm{mg}$; colina, $300 \mathrm{mg}$; biotina, $0.150 \mathrm{mg}$; tiamina, $3.0 \mathrm{mg}$. Premezcla de minerales por kilogramo de alimento: $\mathrm{Fe}, 50.0 \mathrm{mg}$; $\mathrm{Zn}$, $110 \mathrm{mg}$; Mn, $100 \mathrm{mg}$; Cu, $12.0 \mathrm{mg}$; Se, $0.3 \mathrm{mg}$; I, $1.0 \mathrm{mg}$. 


\section{Comportamiento productivo, características de bienestar animal y rendimiento de canal}

El consumo de alimento, ganancia de peso, y conversión alimenticia se registraron desde el día uno hasta el día 42. Al día 43 de edad se seleccionaron al azar 35 aves por tratamiento para evaluar habilidad para caminar, angulación valgus/varus y latencia a postrarse. La habilidad para caminar se evaluó de acuerdo con la metodología descrita por Kestin et $a l^{(13)}$ modificada por Garner et $a l^{(14)}$. La medición se realizó simultáneamente por dos evaluadores que calificaron a cada ave en una escala de 0 a 5 donde: 0. Aves que caminan normalmente; 1. Aves con una ligera dificultad para caminar; 2. Aves con un defecto definido e identificable en su forma de caminar, pero la lesión o daño no obstaculiza el movimiento o el consumo agua y alimento; 3. Aves con un defecto evidente, el cual afecta la habilidad para moverse; 4. Aves con un severo defecto y 5. Aves incapaces de caminar.

La angulación valgus/varus se evaluó de acuerdo con la metodología descrita por Leterrier y $\mathrm{Nys}^{(15)}$. Dependiendo del ángulo de tibia-metatarso, se definieron 4 puntuaciones: 0, pollo normal; 1, pollo con poca angulación (ángulo tibia-metatarso entre 10 y $25^{\circ}$ ); 2 , ave con angulación evidente (ángulo entre 25 y $45^{\circ}$ ) y 3 , angulación severa (ángulo mayor a $45^{\circ}$ ).

Las aves se sometieron a la prueba de latencia a postrarse, según lo descrito por Berg y Sanotra $^{(16)}$. Esta prueba se basa en el contacto corporal del pollo con el agua, que es una experiencia novedosa y adversa para los pollos de engorda. Las aves se colocaron en un recipiente de plástico con agua a $32{ }^{\circ} \mathrm{C}$ a una altura de $3 \mathrm{~cm}$. Se registró el tiempo transcurrido en segundos hasta que cada ave se postró. Si el ave permaneció de pie después de $600 \mathrm{seg}$, la prueba se suspendió. Las aves se evaluaron individualmente sin contacto visual entre ellas.

A los 42 días de edad se seleccionaron al azar siete aves por tratamiento para evaluar el rendimiento de la canal, peso de la pechuga y peso de piernas con muslos. El alimento se retiró $8 \mathrm{~h}$ antes del sacrificio, los pollos se sacrificaron usando un cuchillo aturdidor (modelo VS-200, potencia de entrada $120 \mathrm{~V}-1 \mathrm{~A}$, potencia de salida $50 \mathrm{~V}-0,1 \mathrm{~A}$, Midwest Processing Systems, Minneapolis, MN, EE. UU.), de acuerdo a la Norma Oficial Mexicana NOM-033-SAG/ZOO-2014 ${ }^{(17)}$.

\section{Desarrollo del sistema digestivo y órganos accesorios}

Para evaluar el desarrollo del sistema digestivo se utilizaron los pollos seleccionados para la evaluación del rendimiento en canal. Se midió la longitud del intestino delgado y 
de los ciegos con una cinta métrica y se determinó el peso vacío de proventrículo, molleja, intestino delgado y ciegos, también se obtuvo el peso de hígado, bazo, bolsa de Fabricio, páncreas y corazón. La medición del intestino delgado y de los ciegos se realizó sobre una tela húmeda para evitar se contrajeran.

\section{Análisis estadístico}

Las variables, consumo de alimento, ganancia de peso, y conversión alimenticia se analizaron con un diseño completamente al azar, con un nivel de significancia de 0.05 usando el procedimiento GLM del SAS ${ }^{(18)}$. Las medias de tratamientos se compararon usando la prueba de Tukey ajustada $(P<0.05)$. Las variables, habilidad para caminar y angulación se analizaron con un diseño completamente al azar utilizando los procedimientos GLIMMIX (para datos no paramétricos) y FREC del SAS ${ }^{(18)}$. Los pesos relativos del sistema digestivo, órganos accesorios y latencia a postrarse se analizaron con un diseño experimental completamente al azar con cinco tratamientos y siete repeticiones por tratamiento usando el procedimiento GLM del SAS ${ }^{(18)}$. Las medias de tratamiento se compararon usando la prueba de Tukey y se presentaron como media \pm error estándar.

\section{Resultados}

\section{Comportamiento productivo y rendimiento de canal}

Los pollos de los tratamientos PH y PHMQ tuvieron menor $(P<0.05)$ consumo de alimento y ganancia de peso, respecto a las aves del resto de tratamientos. No se observaron diferencias $(P>0.05)$ entre tratamientos en conversión alimenticia (Cuadro 2 ). En cuanto a las variables de rendimiento de la canal, pechuga y pierna con muslo no hubo diferencias entre tratamientos. 
Cuadro 2: Desempeño productivo de pollos de engorda de 1 a 42 días alimentados con pasta de higuerilla tratada con diferentes métodos de desintoxicación de 1 a 42 días de edad

\begin{tabular}{|c|c|c|c|c|c|c|c|}
\hline \multicolumn{8}{|c|}{ Tratamiento } \\
\hline Variable & PS & $\mathbf{P H}$ & PHA & PHQ & PHAQ & $\mathbf{E E}$ & $P$-valor \\
\hline COA, $\mathrm{g}$ & $4499 \mathrm{a}$ & $3272 \mathrm{~b}$ & $4492 \mathrm{a}$ & $3181 \mathrm{~b}$ & $4575 \mathrm{a}$ & 66.41 & 0.0001 \\
\hline $\mathrm{GP}, \mathrm{g}$ & $2811 \mathrm{a}$ & $1980 \mathrm{~b}$ & $2835 \mathrm{a}$ & $1923 \mathrm{~b}$ & $2835 \mathrm{a}$ & 44.69 & 0.0001 \\
\hline $\mathrm{CA}, \mathrm{g} / \mathrm{g}$ & 1.60 & 1.65 & 1.58 & 1.65 & 1.61 & 0.03 & 0.2322 \\
\hline $\mathrm{RC}, \%$ & 79.96 & 78.31 & 78.91 & 78.03 & 79.91 & 0.66 & 0.1467 \\
\hline $\mathrm{RP}, \%$ & 28.31 & 25.28 & 25.86 & 25.01 & 26.85 & 0.81 & 0.0510 \\
\hline RPM, \% & 20.16 & 21.02 & 21.18 & 20.11 & 20.11 & 0.74 & 0.7002 \\
\hline
\end{tabular}

$\mathrm{PS}=$ testigo, dieta base soya-maíz; $\mathrm{PH}=$ dieta con higuerilla sin desintoxicar; $\mathrm{PHA}=$ dieta con higuerilla tratada con autoclave; $\mathrm{PHQ}=$ dieta con higuerilla tratada con químico; $\mathrm{PHAQ}=$ dieta con higuerilla tratada con autoclave y químico.

$\mathrm{COA}=$ consumo de alimento; $\mathrm{GP}=$ ganancia de peso; $\mathrm{CA}=$ conversión alimenticia; $\mathrm{RC}=$ rendimiento en canal; $\mathrm{RP}=$ rendimiento de pechuga; $\mathrm{RPM}=$ rendimiento de pierna con muslo.

ab Medias de tratamientos con diferentes letras son diferentes $(P<0.05)$. EE=Error estándar.

\section{Habilidad para caminar}

Se encontraron diferencias $(P<0.05)$ por efecto de tratamientos en la habilidad para caminar; los pollos alimentados con las dietas PH y PHMQ mostraron mayor proporción de aves sanas (calificación 0) en comparación con las aves de los otros tratamientos. Aves con calificación 4 y 5 no se observaron en este experimento (Cuadro 3). 
Cuadro 3: Habilidad para caminar, grado de angulación valgus/varus (\%) y latencia a postrarse(s) de pollos de engorda alimentados con higuerilla tratada, de 1 a 42 días de edad

\begin{tabular}{|c|c|c|c|c|c|}
\hline Tratamiento & PS & PH & PHA & PHQ & PHAQ \\
\hline Puntuación & \multicolumn{5}{|c|}{ Habilidad para caminar } \\
\hline 0 & 0.00 & 25.71 & 5.71 & 28.57 & 14.29 \\
\hline 1 & 42.86 & 51.43 & 51.43 & 48.57 & 31.43 \\
\hline 2 & 37.14 & 22.86 & 34.29 & 22.86 & 40.00 \\
\hline 3 & 20.00 & 0.00 & 8.57 & 0.00 & 14.29 \\
\hline 4 & 0.00 & 0.00 & 0.00 & 0.00 & 0.00 \\
\hline 5 & 0.00 & 0.00 & 0.00 & 0.00 & 0.00 \\
\hline$P$-valor & 0.0009 & & & & \\
\hline Puntuación & \multicolumn{5}{|c|}{ Angulación valgus/varus } \\
\hline 0 & 14.29 & 48.57 & 34.29 & 60.00 & 34.29 \\
\hline 1 & 65.71 & 42.86 & 60.00 & 40.00 & 60.00 \\
\hline 2 & 20.00 & 8.57 & 5.71 & 0.00 & 5.71 \\
\hline 3 & 0.00 & 0.00 & 0.00 & 0.00 & 0.00 \\
\hline \multirow[t]{2}{*}{$P$-valor } & 0.0024 & & & & \\
\hline & \multicolumn{5}{|c|}{ Latencia a postrarse } \\
\hline Segundos (s) & 84 & 118 & 103 & 103 & 114 \\
\hline$P$-valor & 0.6681 & & & & \\
\hline
\end{tabular}

\section{Angulación valgus/varus}

Se encontró efecto de tratamientos $(P<0.05)$ en el grado de angulación valgus/varus. La mayor proporción de aves con calificación 0 se encontró en los tratamientos PH y PHMQ y menor proporción de aves con calificación 1. No se observaron pollos con grado de angulación 3 (Cuadro 3).

\section{Latencia a postrarse}

No hubo diferencias $(P>0.05)$ entre tratamientos para latencia a postrarse (Cuadro 3). 


\section{Desarrollo del sistema digestivo y órganos accesorios}

No hubo diferencias $(P>0.05)$ por efecto de tratamientos en el peso relativo del bazo y corazón; sin embargo, el peso relativo del hígado fue menor $(P<0.05)$ en los pollos alimentados con la dieta PS comparados con las aves alimentadas con los tratamientos que incluían pasta de higuerilla. El peso relativo de la bolsa de Fabricio fue menor $(P<0.05)$ en los pollos alimentados con PH y PHMQ, respecto a los pollos con las dietas PS, PHMA y PHMAQ (Cuadro 4). El peso relativo del páncreas, molleja, intestino delgado, y longitud del intestino delgado fueron mayores $(P<0.05)$ en los pollos alimentados con las dietas PH y PHMQ, respecto a PS, PHMA y PHMAQ. El peso relativo de los ciegos de los pollos alimentados con dietas PS, PHA y PHAQ fue menor $(P<0.05)$ con respecto a los pollos del tratamiento PHQ y la longitud del ciego fue mayor $(P<0.05)$ en los pollos alimentados con PH comparado con PHAQ.

Cuadro 4: Peso relativo $(\mathrm{g} / \mathrm{kg})$ y longitud $(\mathrm{cm} / \mathrm{kg})$ de las diferentes secciones del sistema digestivo y órganos accesorios de pollos de engorda alimentados con higuerilla tratada, de1 a 42 días de edad

\begin{tabular}{llllllll}
\hline & \multicolumn{2}{l}{ Tratamientos } & & & & & \\
& PS & PH & PHA & PHQ & PHAQ & EE & $\begin{array}{l}\boldsymbol{P} \text { - } \\
\text { valor }\end{array}$ \\
\hline Bazo & 1.65 & 1.79 & 2.00 & 1.77 & 1.99 & 0.17 & 0.5189 \\
Corazón & 4.08 & 4.38 & 4.15 & 4.35 & 4.26 & 0.12 & 0.3394 \\
Hígado & $18.27 \mathrm{~b}$ & $24.16 \mathrm{a}$ & $21.78 \mathrm{a}$ & $24.34 \mathrm{a}$ & $21.78 \mathrm{a}$ & 0.72 & 0.0001 \\
Bolsa de Fabricio & $1.62 \mathrm{a}$ & $0.70 \mathrm{~b}$ & $1.49 \mathrm{a}$ & $0.81 \mathrm{~b}$ & $1.32 \mathrm{a}$ & 0.08 & 0.0001 \\
Páncreas & $1.63 \mathrm{~b}$ & $2.19 \mathrm{a}$ & $1.82 \mathrm{~b}$ & $2.19 \mathrm{a}$ & $1.50 \mathrm{~b}$ & 0.08 & 0.0001 \\
Proventrículo & $2.94 \mathrm{c}$ & $3.84 \mathrm{ab}$ & $3.35 \mathrm{bc}$ & $4.22 \mathrm{a}$ & $2.94 \mathrm{c}$ & 0.14 & 0.0001 \\
Molleja & $10.50 \mathrm{~b}$ & $14.24 \mathrm{a}$ & $10.50 \mathrm{~b}$ & $14.24 \mathrm{a}$ & $8.61 \mathrm{~b}$ & 0.65 & 0.0001 \\
Intestino delgado & $19.46 \mathrm{~b}$ & $25.17 \mathrm{a}$ & $21.12 \mathrm{~b}$ & $23.60 \mathrm{a}$ & $20.49 \mathrm{~b}$ & 0.56 & 0.0001 \\
Ciegos & $4.91 \mathrm{~b}$ & $5.78 \mathrm{ab}$ & $5.43 \mathrm{~b}$ & $7.13 \mathrm{a}$ & $5.21 \mathrm{~b}$ & 0.39 & 0.0047 \\
Longitud de ciego & $6.36 \mathrm{bc}$ & $7.93 \mathrm{a}$ & $6.65 \mathrm{bc}$ & $7.57 \mathrm{ab}$ & $6.10 \mathrm{c}$ & 0.30 & 0.0007 \\
Longitud intestino & $62.22 \mathrm{~b}$ & $84.19 \mathrm{a}$ & $69.88 \mathrm{~b}$ & $86.50 \mathrm{a}$ & $63.81 \mathrm{~b}$ & 1.95 & 0.0001 \\
\hline
\end{tabular}

$\mathrm{PS}=$ testigo, dieta base soya-maíz; $\mathrm{PH}=$ dieta con higuerilla sin desintoxicar; $\mathrm{PHA}=$ dieta con higuerilla tratada con autoclave; $\mathrm{PHQ}=$ dieta con higuerilla tratada con químico; $\mathrm{PHAQ}=$ dieta con higuerilla tratada con autoclave y químico.

ab Medias de tratamientos con diferentes letras son diferentes $(P<0.05)$. EE=Error estándar. 


\section{Discusión}

La respuesta de los animales alimentados con pasta de higuerilla desintoxicada está determinada por la efectividad del proceso de desintoxicación; de la concentración de la pasta de higuerilla en la dieta; el tiempo de alimentación y la especie animal ${ }^{(19)}$. Se ha registrado en la literatura que los tratamientos con calor aplicados a la pasta de higuerilla disminuyen los compuestos tóxicos, principalmente de la ricina que es la más tóxica, aparentemente altas temperaturas la inactivan ${ }^{(20)}$, estos tratamientos han permitido la inclusión en dietas para pollos de engorda de hasta $10 \%$ sin afectar el comportamiento productivo y el rendimiento de la canal ${ }^{(21,22)}$. En este estudio las aves alimentadas con PHMA y PHMAQ tuvieron un comportamiento productivo similar a las aves alimentadas con PS, esto indica que el calor y la presión en autoclave utilizados en la pasta de higuerilla disminuyeron su toxicidad. Por lo contrario, los pollos alimentados con PH y PHMQ tuvieron un menor consumo y menor ganancia de peso, esto podría atribuirse al contenido de sustancias tóxicas ${ }^{(23)}$. El tratamiento con $\mathrm{Ca}(\mathrm{OH})_{2}$ no disminuyó aparentemente los compuestos tóxicos; los cuales inhiben la síntesis de proteína y afectan principalmente el sistema digestivo, causando descamación y disminución de la longitud de las vellosidades intestinales impidiendo la absorción de los nutrientes y por lo tanto el desarrollo normal de las aves ${ }^{(10,24)}$. El uso de pasta sin desintoxicar en $5 \%$ disminuye el consumo de alimento y la ganancia de peso en pollos de engorda ${ }^{(9,22,25)}$.

No se encontró en la literatura revisada estudios acerca del empleo de la pasta de higuerilla en dietas para pollos de engorda en variables de bienestar animal; sin embargo, en este estudio se encontró que el grado de habilidad para caminar disminuyó en las aves de los tratamientos PS, PHA y PHAQ y la angulación valgus/varus fue mayor en estas aves, esto puede explicarse porque las aves con mayor peso tienen menor habilidad para caminar en comparación con las aves más ligeras ${ }^{(26)}$, pues el peso influye en estas características ${ }^{(27)}$. Los pollos de engorda con mayor peso permanecen postrados más tiempo. En consecuencia, la condición de equilibrio y la angulación de estas aves se ven afectadas, lo que provoca incomodidad al caminar y deterioro en su bienestar $^{(28)}$.

La inclusión de pasta de higuerilla en la dieta produce daños en riñones (inflamación y congestión), agrandamiento del hígado, pulmones inflamados, atrofia de la bolsa de Fabricio y necrosis el bazo ${ }^{(21,29)}$. En el presente estudio, el tamaño del hígado fue mayor en los pollos alimentados con pasta de higuerilla, esto probablemente puede deberse a una mayor actividad metabólica por la presencia de residuos de los compuestos tóxicos ${ }^{(29)}$. Además, se observó un incremento en el tamaño de páncreas, molleja, proventrículo, e intestino en pollos alimentados con PH y PHMQ con respecto al testigo (maíz-pasta de soya). Se han estudiado los pesos de los órganos en otras especies que fueron alimentadas con pasta de higuerilla sin tratar y tratada con hidróxido de calcio en 
la dieta y no se encontraron diferencias en el peso de hígado, corazón riñón y bazo con respecto a la dieta testigo (pasta de soya) ${ }^{(30)}$.

El peso o tamaño de la bolsa de Fabricio es un indicador del estado de inmunocompetencia o inmunosupresión en el ave a nivel de los órganos linfoides ${ }^{(31)}$. La relación del peso de la bolsa de Fabricio con el peso corporal (PBF/PC) puede estar correlacionada con inmunosupresión. Las aves de tres a seis semanas de edad tienen normalmente una relación PBF/PC de 2 a 4, valores de 1 o menor a 1 es indicativo de inmunosupresión y se observa en aves clínicamente enfermas ${ }^{(32)}$. En este estudio la relación PBF/PC de los pollos que fueron alimentados con PH y PHMQ fue menor a 1, lo que indica que probablemente la pasta de higuerilla provocó inmunosupresión en los pollos por la presencia de compuestos tóxicos. Okoye et al ${ }^{(21)}$ observaron una disminución de tamaño en órganos linfoides y necrosis en bolsa de Fabricio en pollos que consumieron una dieta con 10 y $15 \%$ de inclusión de pasta de higuerilla tratada con calor en la dieta.

\section{Conclusiones e implicaciones}

Es posible incluir la pasta de higuerilla desintoxicada con el método de autoclave en dietas de pollos de engorda sin afectar el comportamiento productivo y variables de bienestar. Sin embargo, se desconoce si la carne de estos pollos es apta para el consumo humano, ya que en este estudio no se cuantificaron los residuos de ricina en la carne, por lo que se sugiere realizar estudios para la cuantificación de residuos de ricina en la carne.

\section{Agradecimientos}

La primera autora agradece al Consejo Nacional de Ciencia y Tecnologia (CONACyT) el apoyo financiero para realizar estudios de postgrado, asimismo, al Colegio de Postgraduados Campus Montecillo por la oportunidad brindada.

\section{Literatura citada:}

1. Silva-Lima RL, Severino LS, Silva LMS, Ferreira JJ, Silva VL, Macedo BNE. Substratos para produção de mudas de mamoneira compostos por misturas de cinco fontes de matéria orgánica. Ciênc Agrotec 2006; 30(3):474-479. 
2. Rodríguez RH, Zamarripa CA. Competitividad de la higuerilla (Ricinus communis) para biocombustible en relación a los cultivos actuales en el Edo. de Oaxaca, México. Rev Mex Agr 2013;32:306-318.

3. Azevedo DM, Lima EFO. Agronegócio da mamona no Brasil. Brasília: Embrapa Informação Tecnológica 2001;350.

4. Jiménez OR, Cervantes MR, Vallejo VJA, Rosales RS, Ríos SJC. Perfil de aminoácidos de pastas residuales de piñón tropical (Jatropha curcas) e higuerilla (Ricinus communis). Agrofaz 2011;12(3):173-176.

5. Rostagno HS, Albino LFT, Donzele JL, Gomes PC, Oliveira RF, Lopes DC, et al. Tabelas brasileiras para aves e suínos: composição de alimentos e exigências nutricionais. 2a ed. Viçosa, Brasil: Editora Universitária; 2011.

6. Matos JJB, Días AN, Bueno CFD, Rodríguez PA, Veloso ALC, Faria DE. Metabolizable energy and nutrient digestibility of detoxified castor meal and castor cake for poultry. R Bras Zootec 2011;40(11):2439-2442.

7. Anandan S, Anil KGK, Ghosh J, Ramachandra KS. Effect of different physical and chemical treatments on detoxification of ricin in castor cake. Anim Feed Sci Tech 2004;120:159-168.

8. Oliveira AS, Oliveira MRC, Campos JMS, Machado OLT, Valadares SC, Detmann E, et al. Eficácia de Diferentes Métodos de Destoxificação da Ricina no Farelo de Mamona Conferencia: En: II Congreso de la Red Brasileña de Tecnología y Producción de Biodiesel de 2007. Brasilia.

9. Oso AO, Olayemi WA, Bamgbose AM, Fowoyo OF. Utilization of fermented castor oil seed (Ricinus communis L) meal in diets for cockerel chicks. Arch Zootec 2011;60(229):75-82.

10. Ani AO, Okorie AU. Effects of processed castor oil bean (Ricinus communis L) meal and supplementary dl- methionine on nutrient utilization by broiler chicks. J Anim Plant Sci 2013;23(5):1228-1235.

11.Vázquez JC, Pérez PR. Valores gasométricos estimados para las principales poblaciones y sitios a mayor altitud en México. Rev Inst Nal Enf Resp Mex 2000;13(1):06-13.

12. Aviagen. Ross Broiler Management Manual. Aviagen Ltd., Midlothian, UK. 2017.

13. Kestin SC, Knowles TG, Tinch AE, Gregory NG. Prevalence of leg weakness in broiler chickens and its relationship with genotype. Vet Rec 1992;131:190-194.

14. Garner JP, Falcone C, Wakenel P, Martin M, Mench JA. Reliability and validity of a modified gait scoring system and its use assessing tibial dyschondroplasia in broilers. Br Poultry Sci 2002;43(3):355-363. 
15. Leterrier C, Nys Y. Clinical and anatomical differences in varus and valgus deformities of chick limbs suggest different aetio-pathogenesis. Avian Pathol 1992; 21(3):429-442.

16. Berg C, Sanotra GS. Can a modified latency-to-lie test be used to validate gaitscoring results in commercial broiler flocks. Anim Welf 2003;12(4):655-659.

17. Norma Oficial Mexicana. NOM-033-SAG/ZOO-2014. Métodos para dar muerte a los animales domésticos y silvestres. Diario Oficial de la Federación. México. 26 de agosto de 2015 .

18. SAS Institute, Inc. SAS User's Guide: Statistics version. SAS Institute, Inc. Cary, NC. 2011.

19. Souheila A, Harinder PSM. Potential and constraints in utilizing coproducts of the non-edible oils-based biodiesel industry -an overview. Biofuel co-products as livestock feed Opportunities and challenges. FAO. Rome. 2012.

20. Gardner H, D'Aquin EL, Koltun SP, McCourtney EJ, Vix HLE, Gastrock EA. Detoxification and deallergenization of castor beans. J Amer Oil Chem Soc 1960;37(3):142-148.

21. Okoye JOA, Enunwaonye CA, Okorie AU, Anugwa FOI. Pathological effects of feeding roasted castor vean meal (Ricinus communis) to chicks. Avian Pathol $1987 ; 16(2): 283-290$.

22. Nsa EE, Ukachukwu SN, Akpan IA, Okon B, Effiong OO, Oko OOK. Growth performance, internal organ development and hematological responses of broiler birds fed diets containing different thermal treated castor oil seed meal (Ricinus communis). Glob J Agr Sci 2010;9(2):27-34.

23. Ani AO, Okorie AU. Effects of graded levels of dehulled and cooked castor oil bean (Ricinus communis) meal and supplementary l-lysine on performance of broiler finishers. J Trop Agr Food 2007;6(1):89-97.

24. Audi JMD, Belson MMD, Patel MMD, Schier JMD, Osterloh JMD. Ricin Poisoning. A Comprehensive Review. J Amer Med Assoc 2005;294(18):23422351.

25. Agbabiaka LA, Esonu BO, Madubuike FN. Effect of processing on nutrients and anti-nutrients of castor oil bean (Ricinus communis) seeds and by-products. Pak J Nutr 2011;10(6):561-563.

26. Toscano MJ, Nasr MAF, Hothersall B. Correlation between broiler lameness and anatomical measurements of bone using radiographical projections with assessments of consistency across and within radiographs. Poul Sci 2013;92(9):2251-2258. 
27. Alves MCF, Almeida PICL, Nääs IA, García RG, Caldara FR, Baldo GAA, et al. Equilibrium condition during locomotion and gait in broiler chickens. Rev Bras Cienc Avic 2016;18(3):419-426.

28. Almeida ICL, Garcia RG, Bernardi RIA, Caldara FR, Freitas LW, Seno LO, et al. Selecting appropriate bedding to reduce locomotion problems in broilers. Rev Bras Cienc Avic 2010;12(3):189-195.

29. Mustapha GG, Igwebuike JU, Kwari ID, Adamu SB, Abba Y. The Effect of replacement levels of boiled and fermented castor seed (Ricinus cummunis) meal on the productive performance, nutrient digestibility, carcass characteristics and cost effectiveness in broilers. Int J Sci Nat 2015;6(4):675-682.

30. Gowda NkS, Pal DT, Bellur SR, Bharadwaj U, Sridhar M, Satyanarayana ML, et al. Evaluation of castor (Ricinus communis) seed cake in the total. J Sci Food Agric 2008;89(2):216-220.

31. Cheema MA, Qureshi MA, Havenstein GB. A Comparison of the immune response of a 2001 commercial broiler with a 1957 randombred broiler strain when fed representative 1957 and 2001 broiler diets. Poul Sci 2003;82(10):1519-1529.

32. Giambrone JJ, Clay R. Evaluation of the immunogenicity, stability, pathogenicity, and immunodepressive potential of four commercial live infectious bursal disease vaccines. Poul Sci 1986;65(7):1287-1290. 